\title{
UCRL-TR-214867
}

LAW RENCE LIVERMORE N A T IO N A L LABORATORY

\section{ASC Supercomputers Predict Effects of Aging on Materials}

A. Kubota, D. B. Reisman, W. G. Wolfer

August 29, 2005 
This document was prepared as an account of work sponsored by an agency of the United States Government. Neither the United States Government nor the University of California nor any of their employees, makes any warranty, express or implied, or assumes any legal liability or responsibility for the accuracy, completeness, or usefulness of any information, apparatus, product, or process disclosed, or represents that its use would not infringe privately owned rights. Reference herein to any specific commercial product, process, or service by trade name, trademark, manufacturer, or otherwise, does not necessarily constitute or imply its endorsement, recommendation, or favoring by the United States Government or the University of California. The views and opinions of authors expressed herein do not necessarily state or reflect those of the United States Government or the University of California, and shall not be used for advertising or product endorsement purposes.

This work was performed under the auspices of the U.S. Department of Energy by University of California, Lawrence Livermore National Laboratory under Contract W-7405-Eng-48. 


\title{
ASC Supercomputers Predict Effects of Aging on Materials
}

\author{
Alison Kubota, David B. Reisman, and Wilhelm G. Wolfer \\ Lawrence Livermore National Laboratory
}

\section{Executive Summary}

In an extensive molecular dynamics (MD) study of shock compression of aluminum containing such microscopic defects as found in aged plutonium, LLNL scientists have demonstrated that ASC supercomputers live up to their promise as powerful tools to predict aging phenomena in the nuclear stockpile. Although these MD investigations are carried out on material samples containing only about 10 to 40 million atoms, and being not much bigger than a virus particle, they have shown that reliable materials properties and relationships between them can be extracted for density, temperature, pressure, and dynamic strength. This was proven by comparing their predictions with experimental data of the Hugoniot, with dynamic strength inferred from gas-gun experiments, and with the temperatures behind the shock front as calculated with hydro-codes. The effects of microscopic helium bubbles and of radiation-induced dislocation loops and voids on the equation of state were also determined and found to be small and in agreement with earlier theoretical predictions and recent diamond-anvil-cell experiments. However, these microscopic defects play an essential role in correctly predicting the dynamic strength for these nano-crystalline samples. These simulations also prove that the physics involved in shock compression experiments remains the same for macroscopic specimens used in gas-gun experiments down to micrometer samples to be employed in future NIF experiments. Furthermore, a practical way was discovered to reduce plastic instabilities in NIF target materials by introducing finely dispersed defects.

\section{Technical Accomplishments}

Aluminum has been chosen as a model material because reliable embedded atom potentials $[1,2]$ are available, it has a low melting point, and an exhaustive analysis of Hugoniot data and comparison with first-principle EOS calculations has recently been carried out [3]. A typical snapshot from one of the many MD simulations is shown in

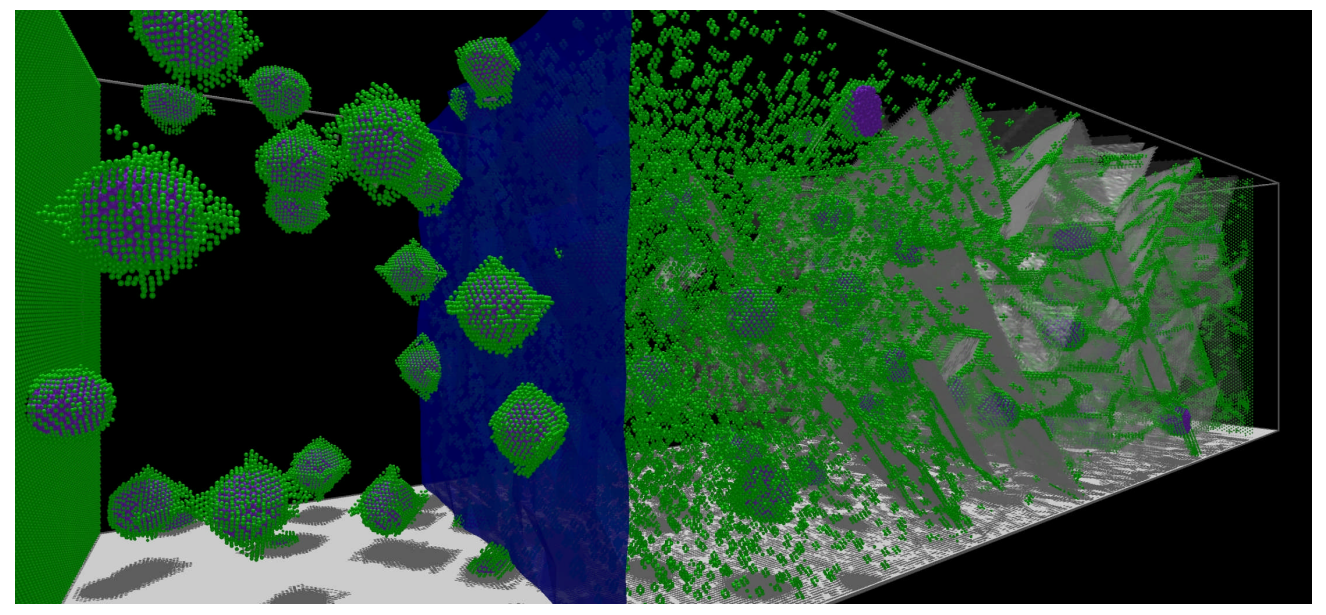

Figure 1. Shock compression at $12 \mathrm{GPa}$ of Aluminum with Helium Bubbles. 
Figure 1. One sees an aluminum sample containing helium bubbles with sizes and number densities similar to what has been found in aged plutonium. A piston has impacted the rear surface, sending a strong, planar shock wave to the front. Its position at time 15 ps after impact is identified by the blue surface. At the maximum impact force of $12 \mathrm{GPa}$ employed in this simulation, a profuse generation and multiplication of dislocations is observed, originating from the bubble surfaces. This dislocation activity is extremely complex, and a detailed dissection of all the dislocation reactions appears to be a daunting task. Similar pictures have been obtained in MD simulations by other researchers in the past, and the difficulties in relating their content to macroscopically measurable parameters has been duly noted.

What distinguishes the present study from previous ones is the extensive post-processing of the atomistic data. It consists of a fine-grained determination of the spatial and temporal distributions for the material density, the pressure, all stresses, all strains, and for the temperature. An example is shown in Figure 2.

Instead of extracting the EOS from the relationship between particle and shock velocities, as traditionally done in shock-compression experiments, one can now pair up directly a local density with its local pressure. Taking regions where both attain their maximum values, indicating equilibrium states, Figure 3 shows the Hugoniot from simulations performed with piston pressures of 4, 6, 12, 20, and $30 \mathrm{GPa}$. This figure also contains results from simulations on aluminum without any initial defects, with small dislocation loops, or with voids. In addition, it compares it with the best Hugoniot for aluminum from Ref. [3].

The temperatures reached behind the shock are shown in Figure 4, and are compared with the predictions from a hydro-code with a strength model for aluminum. The von Mises stress, or the equivalent stress, has been found to reach a nearly uniform minimum value behind the shock front for samples with initial, microscopic defects. These minimum values, obviously the dynamic strength values of the material, are shown in Figure 4 as a function of the applied piston pressure. The dynamic strength values obtained from the computer simulations fall within a band from about 1-1.5 GPa, nearly independent of applied pressure or type of microscopic defects. Similar values have been measured recently by Huang and Asay [4].

\section{Perspective and Outlook}

The surprising success of MD simulations as a viable computational approach to shock physics needs to be evaluated in the broader context of high-performance computing for the stockpile stewardship mission. This evaluation is different and specific for the EOS and for the dynamic strength.

Equation of State: The established theoretical and computational approach to the equation of state of solids is to divide the pressure into three separate contributions. The cold pressure, the thermal pressure, and the electronic pressure. The first is obtained from first-principle calculations using various implementations of the density functional theory 
(DFT). The second is obtained from phonon calculations or more often from the simpler Mie-Grüneisen model. The electronic contribution is based on Thomas-Fermi models for excited states of the electron gas at elevated temperatures. The latter contribution is negligible for solids, where the dominant contribution comes from the cold pressure.
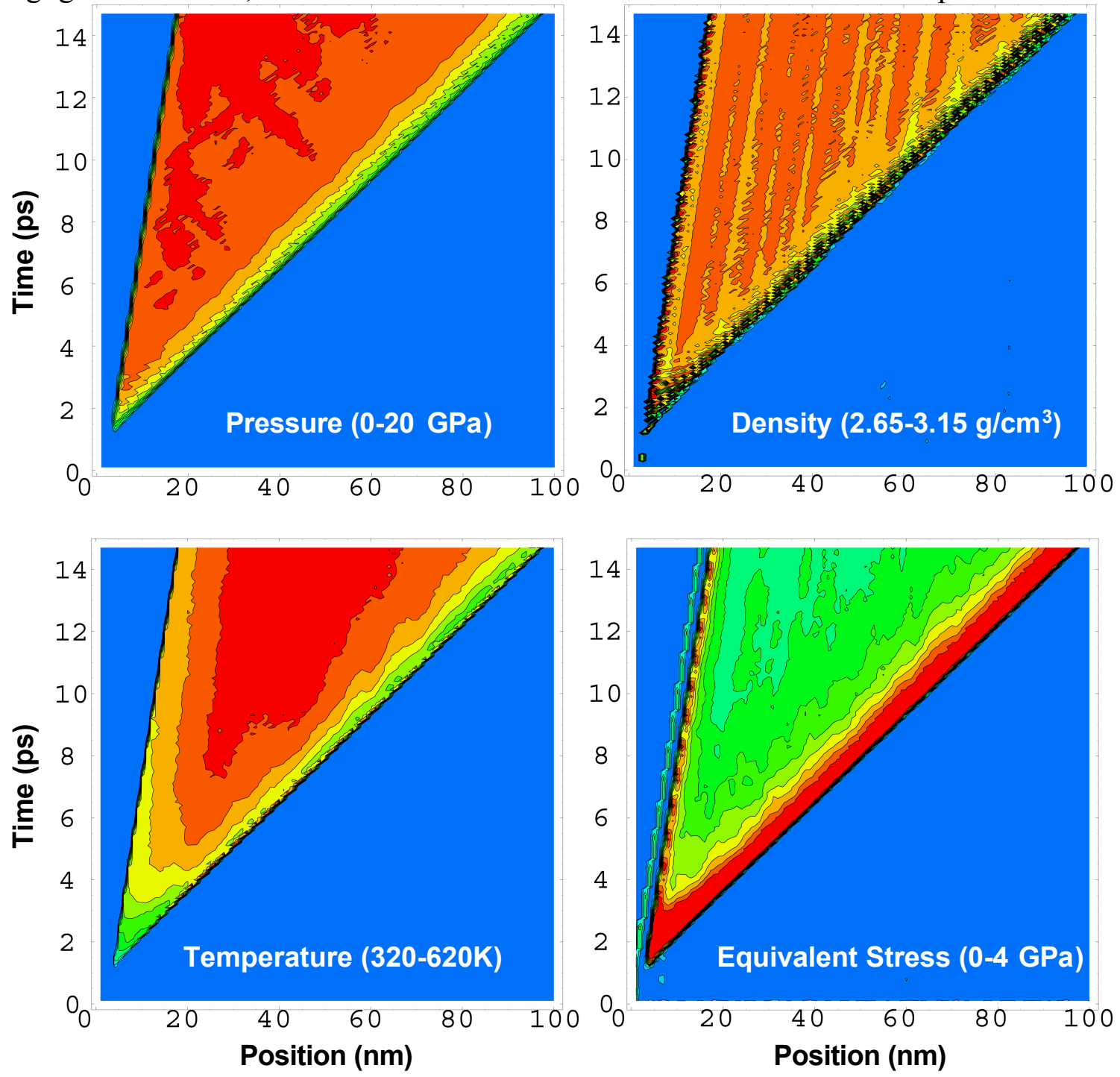

Figure 2. The spatio-temporal distributions of the mean stress or pressure (top left), of the density (top right), of the temperature (bottom left), and of the equivalent stress (bottom right) in an aluminum sample with helium bubbles (helium content is $2.3 \%$ and would correspond to that found in 580 year old $\mathrm{Pu}$ ) impacted on the left with a piston pressure of $20 \mathrm{GPa}$. Hues from blue to red map linearly the range of values indicated in each figure. The slope of the triangular section on the left is the inverse of the particle velocity, and the slope on the right is the inverse of the shock velocity. Sections through these distributions at a given time after the impact (which occurred at $1 \mathrm{ps}$ ) give the spatial distributions through the compressed portion of the sample. For example, at time $t$ $=12 \mathrm{ps}$, the shock front has advanced a distance of $80 \mathrm{~nm}$ (in the Lagrangian frame). The temperature rises rapidly and reaches its maximum value of $610 \mathrm{~K}$ at a distance of $20 \mathrm{~nm}$ 
behind the shock front. The equivalent stress that drives plastic deformation peaks at the shock front and then precipitously drops behind it and reaches a minimum value, which is the dynamic strength of the material for the imposed piston pressure.

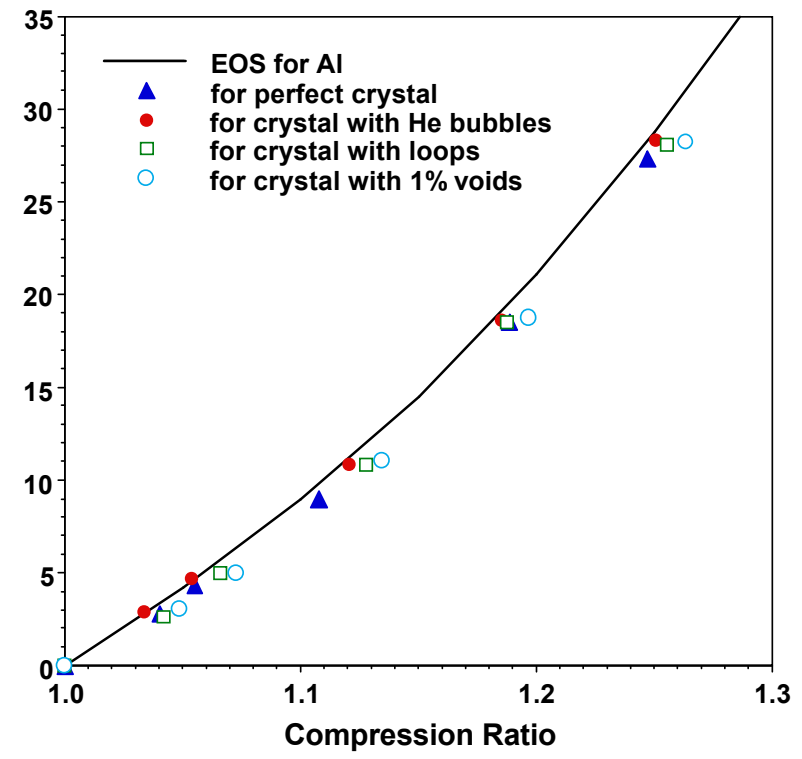

Figure 3. The Hugoniot of Al with and without defects, and its comparison with the best available data.

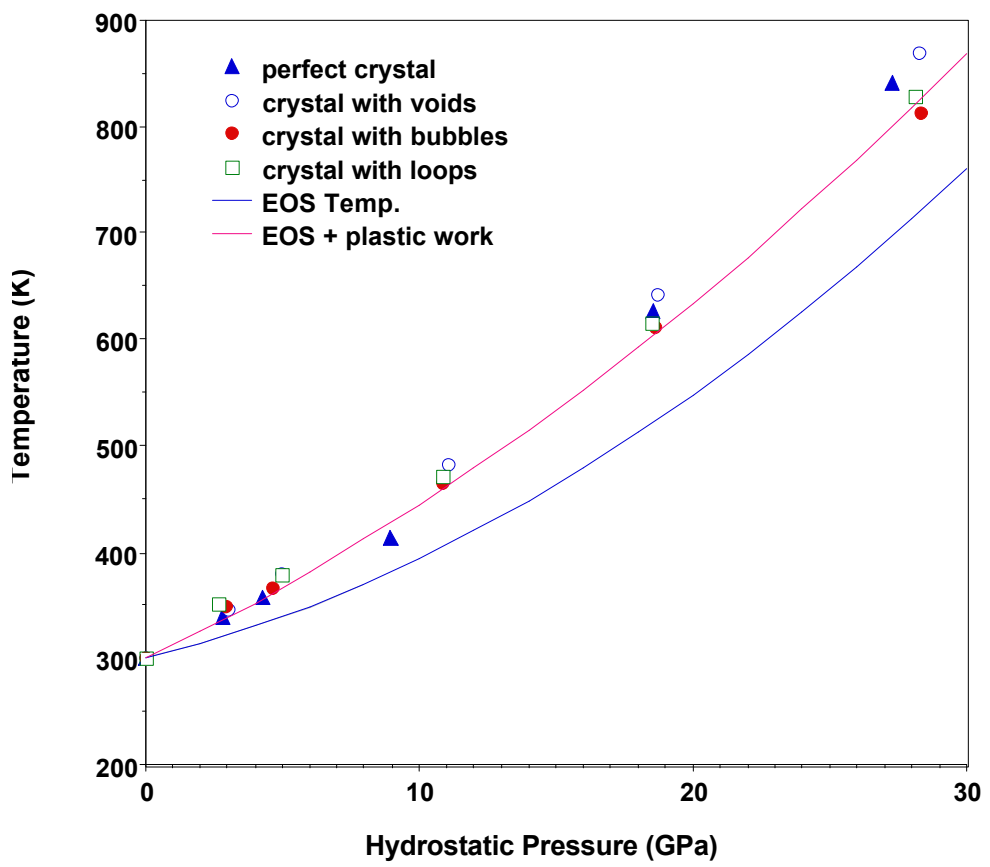

Figure 4. The equilibrium temperature reached behind the shock front in Al. The curves are values calculated with a hydro-code with and without the heating from plastic deformation. 


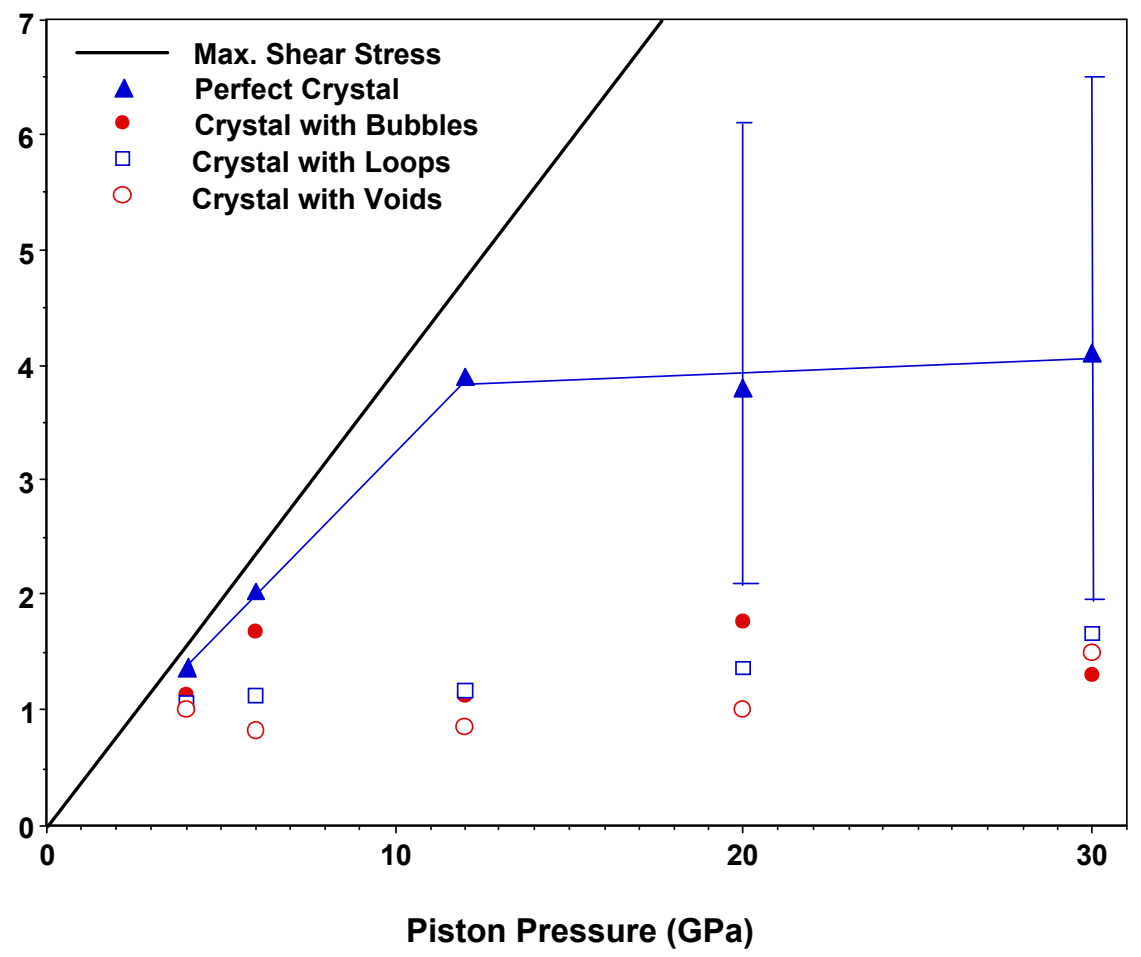

Figure 5. The dynamic strength of Al with and without defects. The maximum equivalent stress at the shock front is the bold line. Al without initial defects exhibits inhomogeneous plastic deformation, and the equivalent stress varies through the compressed portion of the samples over the range indicated by the vertical bars.

However, the DFT predictions for the cold pressure must often be adjusted slightly to better match experimental data [3]. Classical MD relies on semi-empirical potentials (EAM or MEAM) which are fitted to DFT force calculations, and measured parameters such as lattice constants, elastic moduli, their pressure derivatives, heat of sublimation, vacancy formation energy, liquid structure factors, etc. If a reliable potential is obtained in this way, classical MD will evaluate the combined cold and thermal pressure with an accuracy that matches the established theoretical approach. The results in Figure 3 show that the MD simulations give indeed a Hugoniot which is only slightly different than the best Hugoniot results [3]. By a minor modification of the EAM potential, being the analog of adjusting DFT predictions, excellent agreement can be achieved. The classical MD approach provides additional advantages because the system size can now be increased dramatically from hundreds to billions of atoms. This enables the investigation of EOS for solids with defects, or composed of multiple phases. Furthermore, it is now possible, as shown in this study, to define and compute thermodynamic variables such as temperature, density, pressure, and entropy production on a local and global scale. This is still beyond the reach of quantum molecular dynamics methods.

Dynamic Strength: The multi-scale approach to dynamic strength consists of four methods: DFT calculations are employed to determine the inner core structure of 
dislocations; next, atomistic simulations with semi-empirical potentials investigate the basic reactions of dislocations with other defects; at the third level, the forces that set in motion a particular dislocation segment are evaluated from the internal stresses produced by all other dislocation segments and all other defects present, and finally from the applied stresses. The latter are usually determined from finite element calculations using macroscopic plasticity models.

The results of this study demonstrate that the first three methods of the multi-scale approach are all covered by classical MD, at least in the context of strong shock deformation. Post-processing methods developed in this study can analyze in great detail the Burgers vectors and the dislocation reactions within the microstructure produced behind the shock front. However, a more direct approach that accomplishes the necessary averaging over the complex dislocation activity has been found and developed. It consists of computing the thermodynamic variables as well as the state of local stress and strain. Dislocation plasticity in crystals without pre-existing defects or compressed at low strain rates was found to be highly non-uniform. To simulate this type of heterogeneous deformation or flow localization requires then simulation samples larger than 40 million atoms. They are, however, within reach of the new generation of supercomputers, such as BGL.

This work was performed under the auspices of the U. S. Department of Energy by the University of California, Lawrence Livermore National Laboratory under Contract No. W-7405-Eng-48. This project 05-ERD-020 was funded by the Laboratory Directed Research and Development Program at LLNL. We thank the Lawrence Livermore National Laboratory MCR, Thunder, and BG/L teams for the generous allocation of computer resources used to perform this work.

\section{References}

1. Voter, A. F., "Embedded Atom Method potentials for seven FCC metals: Ni, Pd, Pt, $\mathrm{Cu}, \mathrm{Ag}, \mathrm{Au}$ and Al," Los Alamos Unclassified Technical Report \#LA-UR 93-3901, 1993.

2. Mundy, C. J., "Al-He potential from Car-Parrinello molecular dynamics," unpublished results.

2. Chisolm, E. D. et al., "Test of a theoretical equation of state for elemental solids and liquids," Physical Review B, Vol. 68, 104103, 2003.

4. H. Huang and J.R. Asay, "Compressive strength measurements in aluminum for shock compression over the stress range of 4-22 GPa.", accepted for publication in J. Appl. Phys. 